\title{
Sequential dependencies and response range in cross-modality matches of duration to loudness
}

\author{
LAWRENCE M. WARD \\ University of British Columbia, Vancouver, British Columbia, Canada V6T 1W5
}

\begin{abstract}
Subjects matched durations of keypresses to loudnesses of pure tones. Resulting duration responses were found to be assimilated to the value of the immediately preceding stimulus and responses from one to at least six trials back in the sequence of loudness stimuli. Responses were contrasted with the values of stimuli two through six trials back in the sequence. These sequential dependencies and other properties of the data were predicted by a cognitive model in which cross-modality matches are mediated by category judgments of stimuli on both continua, and subjects use heuristic strategies to reduce response uncertainty.
\end{abstract}

Power functions relating responses to stimuli in psychophysical judgment experiments have been said to reflect the operating characteristics of sensory transducers (Stevens, 1961). Neurophysiological data are at present equivocal as to the origin of power functions governing subjective magnitude, and as to the correct interpretation of their exponents (see, e.g., Lipetz, 1969. and Stevens, 1970). Thus, much of the evidence for the operating characteristic interpretation of power functions must still come from behavioral experiments. Particularly impressive (although not conclusive) in this regard has been the demonstration of consistency of power functions fitted to cross-modality matching data with those fitted to magnitude estimation data (cf. Stevens, 1969). The argument has been made that in cross-modality matching the subject is matching one raw sensation to another, and thus is matching the operating characteristics of the two sensory transducers involved. Cross-modality matching is said to be a "pure" psychophysical technique uncontaminated by number or other biases known to afflict other forms of psychophysical judgment.

Surprisingly, in view of the obvious importance of the cross-modality matching technique to the validation and production of scales of measurement (see, also. Krantz, 1972), very little has been done in the way of investigating the various properties of cross-modality matching data. In particular, I know of no reports of direct investigations of these data for common psychophysical biases such as sequential dependencies or time order error, which have been observed in many other judgment situations. The case of sequential dependencies is especially interesting. since not only have they been demonstrated in absolute category judgments (Ward, 1972) but also in magnitude estimations (Ward, 1973) which have been

This research was supported by a Biomedical Science Support Grant from NIH via Rutgers. and a Rutgers University Summer Research Fellowship. said to be a unique form of cross-modality matching in which stimulus intensity is matched to "number intensity" (cf. Stevens, 1966). Indeed, several authors have suggested that all psychophysical judgments (including "pure" cross-modality matching) are based on a matching operation which is constrained differently in different tasks (Stevens, 1966; Ward, 1973). If this is true, then it is reasonable to expect cross-modality matching data to behave similarly to other psychophysical data, particularly in sequential dependencies.

A somewhat more precise statement of the above arguments follows in the form of an informal process model of cross-modality matching. The model (which I will call the cognitive model) is a three-stage decision model based on the model proposed by Ward and Lockhead (1971) for category judgments without feedback. In the present extension, the subject in a cross-modality matching experiment is assumed to have available in long-term memory (LTM) a (self-constructed) category scale for each of the perceptual continua used in the experiment. The two scales are assumed constrained to have the same number of categories, determined by the number easily used on the noisiest continuum (probably about 5-9).

In Stage 1 of the model, a stimulus presentation on continuum 1 is assumed to give rise to an internal representation of the stimulus [held in short-term memory (STM)] which is compared to the continuum 1 category scale. A rough match between the stimulus representation and an area of the category scale is determined by successively comparing the stimulus representation to internally generated representations of the various areas of the scale. The width of the matching area (an "indifference" region) depends on factors such as signal-to-noise ratio, the precision of the representations involved, etc. Generally. the more stimulus uncertainty, the more response uncertainty. i.e. the more categories in the "indifference" region. If response uncertainty exists, then a single response 
category is chosen from those in the "indifference" region via one or both of at least two heuristic response strategies (discussed later).

Stage 2 of the process is simply a translation of the response category finally chosen on the continuum 1 category scale in Stage 1 , into the corresponding category on the continuum 2 category scale. This is a simple one-to-one mapping procedure, assumed not to be subject to systematic errors.

Stage 3 is equivalent to a category production operation which is the reverse of Stage 1 , with the exception that this operation is assumed to be unbiased. Here, the subject is assumed to adjust the amplitude of the continuum 2 stimulus so that a rough match is achieved between the internal representation of that stimulus and the internal representation of the area of the continuum 2 category scale selected by Stage 2 .

Thus, in the cognitive model outlined above, a tone judged to be "quite loud" via Stage 1 would be mapped to the corresponding response continuum category [say "quite long" (in duration)] via Stage 2, and a duration representative of "quite long" then produced via Stage 3. Numerical category labels could also be used, and would facilitate the process.

Ward and Lockhead (1971) have shown how the use of two heuristic strategies to reduce response uncertainty in category judgments can give rise to systematic biases in those data. Ward and Lockhead (1971) argue that contrast between the response to a stimulus and the immediately previous five or six stimulus values is produced by subjects' attempting to use the available response categories equally often over the local (5-6 trials-STM span-see Simon, 1974) random sequence of stimuli. To do this, they may shift the entire "indifference" region on a particular trial away from the area of the response scale in which most of the preceding five or six responses (or stimuli) occurred, producing contrast with those responses (or stimuli). Ward and Lockhead (1971) also argued that assimilation between a response and the immediately preceding response or stimulus value can be produced by choosing a category in the "indifference" region which is nearer the immediately previous stimulus (or response) more often than one farther from that stimulus (or response). Subjects may do this if they have observed that small distances between stimuli adjacent in a random sequence occur more frequently than do large ones, and they are attempting to maximize with respect to this fact. Both strategies would thus affect the frequency distribution of responses to the local sequence of stimuli, the first by shifting the entire distribution (and thus its mean) away from previous frequently used responses (and stimuli), and the second by skewing the distribution away from the immediately previous response (and stimulus) (but not enlarging the "indifference" region) and thus shifting the mean toward that response (and stimulus). If both strategies were used by a subject. they would of course clash, with the larger assimilation effect (found so in several studies) obscuring any first-order contrast. Compounding of the assimilation might lead to higher order assimilation which would be progressively weaker (Ward \& Lockhead, 1971), and the contrast effect would grow more reliable as the competing compounded assimilation effect grew weaker. Just this pattern of reliable first-order assimilation and progressively stronger higher order contrast (up to about six trials back in the sequence) was observed in category judgments by Ward and Lockhead (1971) and $W$ ard (1972).

Since Stage 1 of the informal model of cross-modality matching outlined above consists of category judgments without feedback, to the extent that these judgments are affected by the biases just described, these biases should be reflected in cross-modality matches if they are produced by processes similar to those of the cognitive model. Thus, the cognitive model predicts sequential dependencies in cross-modality matching data, although finding such effects is not sufficient to establish the model. The present paper reports a study of cross-modality matching of duration to loudness in an experimental situation designed to be optimal for such judgments and similar to previous studies of cross-modality matching. The data were investigated for the presence of the predicted sequential dependencies. In addition, several other properties of the data were discussed, including the prediction of the cognitive model that response range should correspond to the number of categories that can be easily dealt with (five to nine categories, or channel capacity) on the continua involved.

\section{METHOD}

Ten paid volunteer subjects (students at Rutgers University) were presented with a series of pure tones and were asked to press a telegraph key for a duration which they believed matched the loudness of each pure tone. In this manner, each subject matched 500 loudnesses after he matched three practice loudnesses (the same for all subjects). The loudness stimuli were 500 -msecduration, $1,000-\mathrm{Hz}$ sinusoids generated by a Hewlett-Packard 200-CD oscillator and amplified and gated by a Grason-Stadler electronic switch. A tape reader selected one of 10 different potentiometers on each trial, and the resulting amplitude was delivered diotically through Superex St-S headphones to the subject, who was seated in an IAC sound-attenuation chamber. The loudness stimuli were presented in one of five different random orders (randomized over the entire series of 500 stimuli). Each order was used for two subjects. There were no differences in the results as a function of order of stimuli. The amplitudes used were $1.46,2.59,4.61,8.19,14.60,25.90,46.10,81.90,146.0$, and $259.0 \mathrm{mV}$. Each occurred 50 times in each random order. The softest stimulus was about $49 \mathrm{~dB}$ re $.0001 \mathrm{dynes} / \mathrm{cm}^{2}$, and the total stimulus range was from 49 to $94 \mathrm{~dB}$ in 5-dB steps.

Subjects' responses (durations of keypress) were measured with an electronic timer ${ }^{1}$ accurate to $1 \mathrm{msec}$. Each stimulus in the random order was presented about $3 \mathrm{sec}$ after the end of the 
subject's response to the previous stimulus. and each subject's data were collected in one continuous session. which averaged $1 \mathrm{~h}$ in length. After responding to each of the 500 loudnesses, subjects also provided 15 intramodal matches of duration of keypress to five each of three different durations of tone (a $14.60-\mathrm{mV}$ tone of $500,1,000$. or $5.000 \mathrm{msec}$ ) in different random orders. to check their accuracy in reproducing memories of durations.

\section{RESULTS}

\section{Intramodal Duration Matching}

All subjects performed adequately on the intramodal duration matching task. That is, their average matches of the different durations were clearly different. indicating that they could discriminate among durations in the same range as those produced as responses to the loudness stimuli. The mean matches for the 500-, 1.000-, and 5.000-msec-duration tones were 929, 1,550, and $4.498 \mathrm{msec}$, respectively, with average standard errors of the mean of 104,161 , and $259 \mathrm{msec}$, respectively. It can be seen that the average matches are clearly different in terms of the average standard errors; this was also true of the data of each individual subject. Notice that the variability of the duration matches increases with increasing duration. This is a common finding in such data (cf. Woodrow, 1951).

\section{Common Modulus}

Since no modulus was specified in this experiment, it was necessary to transform all of the data to the same modulus whenever they were averaged across subjects. This was done by solving the equation

$$
\left[\prod_{i=1}^{n} \mathrm{KX}_{\mathrm{i}}\right]^{1 / \mathrm{n}}=1,000
$$

for $K$ for the $n(=50)$ responses $(X)$ of each subject to the $14.60-\mathrm{mV}$ loudness. and then multiplying all of each subject's responses by his own $\mathbf{K}$. This had the effect of adjusting the subject's responses so that the geometric mean response to the $14.60-\mathrm{mV}$ stimulus was $1.000 \mathrm{msec}$. Hereafter, whenever a common modulus is indicated, this procedure was used.

\section{Sequential Dependencies}

Tables 1 and 2 show the results of analyses of the transformed (to a common modulus) cross-modality matches for the sequential dependencies predicted by the cognitive model outlined in the introduction. The entires in Tables 1 and 2 are the means of the rows of the $S(N-K)$ (stimulus on Trial $N-K)$ or $R(N-K)$ by $\mathrm{S}(\mathrm{N})$ matrix of geometric mean responses (durations) on Trial $\mathrm{N}$ over all subjects, as a function of the stimulus (Table 1) or response (Table 2$)^{2}$ on Trial $\mathbf{N}-\mathbf{K}$. Of interest here are the rank-order correlation coefficients between $S(N-K)$ or $R(N-K)$ and the overall average response on Trial $N$. A positive correlation indicates assimilation of responses to the previous stimulus or response value; a negative correlation indicates contrast. The size of the correlation indicates only the reliability of the relationship. The magnitude of the sequential dependencies will be precisely expressed later, using a formulation of Cross (1973). Tables 1 and 2 display both the correlations calculated from the tabled data and the average correlations calculated from the analogous tables for the individual subjects. As can be seen. the averages of the individual correlations qualitatively mirror the correlations calculated from the pooled data, although in general they are much smaller. Correlations for individual subjects seldom reached statistical significance (for example, for $K=1$, only two subjects in the stimulus analysis and four in the response analysis had significant correlations, $\alpha=.05$ one-tailed) but for most subjects were of the same sign as the pooled correlations (for example, for $K=1.8$ of 10 subjects in the stimulus

Table 1

Average Responses on Trial $N$ as a Function of the Stimulus on Trid $N-K(K=1,10)^{*}$

\begin{tabular}{|c|c|c|c|c|c|c|c|c|c|c|}
\hline \multirow{2}{*}{$\begin{array}{c}\text { Stimulus } \\
\text { on Trial } \\
\mathbf{N}-\mathbf{K}\end{array}$} & \multicolumn{10}{|c|}{ Average Response on Trial $\mathrm{N}$} \\
\hline & $\mathbf{K}=1$ & $K=2$ & $K=3$ & $K=4$ & $K=5$ & $K=6$ & $\dot{K}=7$ & $K=8$ & $K=9$ & $K=10$ \\
\hline 1 & 1007 & 1073 & 1060 & 1077 & 1100 & 1072 & 1073 & 1078 & 1076 & 1073 \\
\hline 2 & 1002 & 1058 & 1069 & 1075 & 1068 & 1036 & 1039 & 1073 & 1037 & 1024 \\
\hline 3 & 1048 & 1069 & 1089 & 1065 & 1060 & 1059 & 1084 & 1064 & 1049 & 1078 \\
\hline 4 & 1077 & 1062 & 1097 & 1066 & 1074 & 1083 & 1100 & 1070 & 1083 & 1073 \\
\hline 5 & 1053 & 1082 & 1078 & 1068 & 1081 & 1067 & 1060 & 1047 & 1070 & 1063 \\
\hline 6 & 1082 & 1063 & 1046 & 1054 & 1076 & 1085 & 1067 & 1064 & 1074 & 1072 \\
\hline 7 & 1078 & 1043 & 1058 & 1066 & 1046 & 1066 & 1084 & 1087 & 1070 & 1094 \\
\hline 8 & 1054 & 1045 & 1028 & 1051 & 1026 & 1058 & 1025 & 1046 & 1046 & 1064 \\
\hline 9 & 1102 & 1068 & 1066 & 1056 & 1055 & 1049 & 1081 & 1073 & 1078 & 1051 \\
\hline 10 & 1089 & 1040 & 1018 & 1028 & 1028 & 1022 & 1034 & 1037 & 1059 & 1070 \\
\hline$\frac{\mathbf{r}^{\prime}}{\mathrm{r}_{\mathrm{I}}^{\prime}}$ & $\begin{array}{l}.857 \\
.280\end{array}$ & $\begin{array}{l}-.539 \\
-.145\end{array}$ & $\begin{array}{l}-.612 \\
-.102\end{array}$ & $\begin{array}{l}-.955 \\
-.164\end{array}$ & $\begin{array}{l}-.721 \\
-.198\end{array}$ & $\begin{array}{l}-.406 \\
-.192\end{array}$ & $\begin{array}{l}-.294 \\
-.088\end{array}$ & $\begin{array}{l}-.424 \\
-.167\end{array}$ & $\begin{array}{r}.021 \\
-.072\end{array}$ & $\begin{array}{r}-.142 \\
.032\end{array}$ \\
\hline
\end{tabular}

${ }^{*}$ Means of rows of $S(N-K)$ by $S(N)$ matrix of geometric mean responses (milliseconds). Two-tailed $r_{\text {crit }}^{\prime}$ for .10 level is \pm .564 , for .05 level is \pm .648 , for .01 level is \pm .818 . $\vec{r}_{1}^{t}$ is the average of the $r^{\prime}$ sor the data of the individual subjects. 
Table 2

Average Responses on Trial $N$ as a Function of the Response "Bin" on Trial $N-K(K=1,10) *$

\begin{tabular}{|c|c|c|c|c|c|c|c|c|c|c|}
\hline \multirow{2}{*}{$\begin{array}{c}\text { Response } \\
\text { on Trial } \\
\mathbf{N}-\mathbf{K} \\
\end{array}$} & \multicolumn{10}{|c|}{ Average Response on Trial $\mathbf{N}$} \\
\hline & $K=1$ & $K=2$ & $K=3$ & $K=4$ & $K=5$ & $K=6$ & $\mathrm{~K}=7$ & $\mathrm{~K}=8$ & $K=9$ & $K=10$ \\
\hline 1 & 1108 & 1157 & 1162 & 1179 & 1186 & 1194 & 1187 & 1185 & 1173 & 1184 \\
\hline 2 & 1146 & 1155 & 1170 & 1156 & 1140 & 1129 & 1114 & 1154 & 1174 & 1129 \\
\hline 3 & 1179 & 1202 & 1194 & 1191 & 1221 & 1183 & 1212 & 1216 & 1203 & 1255 \\
\hline 4 & 1182 & 1203 & 1234 & 1204 & 1141 & 1190 & 1249 & 1195 & 1204 & 1176 \\
\hline 5 & 1264 & 1240 & 1246 & 1206 & 1202 & 1220 & 1214 & 1171 & 1237 . & 1250 \\
\hline 6 & 1284 & 1273 & 1288 & 1274 & 1358 & 1338 & 1276 & 1290 & 1314 & 1343 \\
\hline 7 & 1208 & 1214 & 1193 & 1239 & 1204 & 1234 & 1226 & 1244 & 1268 & 1228 \\
\hline 8 & 1254 & 1269 & 1245 & 1282 & 1283 & 1248 & 1256 & 1239 & 1237 & 1257 \\
\hline 9 & 1247 & 1206 & 1242 & 1214 & 1203 & 1222 & 1203 & 1217 & 1198 & 1218 \\
\hline 10 & 1275 & 1238 & 1214 & 1229 & 1235 & 1226 & 1221 & 1232 & 1229 & 1223 \\
\hline $\mathbf{r}^{\prime}$ & .758 & .673 & .503 & .794 & .612 & .709 & .467 & .660 & .512 & .358 \\
\hline$\overline{\mathbf{r}}_{\mathrm{I}}^{\prime}$ & .383 & .334 & .327 & .234 & .238 & .346 & .238 & .228 & .239 & .196 \\
\hline
\end{tabular}

* Means of rows of $R(N-K)$ by $S(N)$ matrix of geometric mean responses (milliseconds). Two-tailed $r^{\prime}$ crit for .10 level is \pm .564 , for .05 level is \pm .648 , for .01 level is $\pm .818 . \bar{r}_{I}^{\prime}$ is the average of the $r^{\prime}$ s for the data of the individual subjects.

analysis and 9 of 10 in the response analysis had positive correlations, and none of the three negative correlations were statistically significant). Thus, the pooled data can be considered representative of the data of most of the individual subjects.

Table 1 indicates that there is assimilation of the (transformed) duration matches to the loudness level on the immediately preceding trial. There appears to be weak contrast to stimuli two and three trials back in the sequence, and stronger contrast to stimuli on Trials $\mathrm{N}-4$ and $\mathrm{N}-5$. Thereafter, there seem to be no reliable sequential dependencies. Table 2 indicates reliable assimilation not only to the response "bin" on Trial $\mathrm{N}-1$, but also to those on Trials $\mathrm{N}-2, \mathrm{~N}-4$, $\mathrm{N}-5, \mathrm{~N}-6$, and $\mathrm{N}-8 \quad(\alpha=.05$ one-tailed $)$ and weaker assimilation on the remainder of the trials up to 10 back in the sequence. The contrast found in the stimulus analysis is not present.

These dependencies are highly similar to those reported by Ward (1972) for category judgments without feedback, and are also of the same form as those reported by Ward and Lockhead (1971) for guesses of which of 10 digits would occur next in a random sequence. The one noticeable difference is that higher order contrast effects do not appear in the present data in the response analysis, as they do in that analysis in previous studies of category judgment and number guessing. The present data are also somewhat different from dependencies found in magnitude estimation data (Ward, 1973), in which higher order contrast also is not present in the stimulus analysis (a response analysis was not reported by Ward, 1973). All dependencies in magnitude estimations are assimilative, with higher order effects apparently caused by compounding of the strong first-order effect (cf. Ward \& Lockhead, 1971). This may also be the explanation for the lack of contrast effects in the response analysis of the present data, i.e., the compounded assimilation effects cancel out the weaker contrast effects. The overall pattern of the dependencies discovered, however, is similar enough to that found in category judgments to assert that the prediction of the cognitive model is verified.

\section{Power Law and Response Range}

A power function of the form

$$
\mathrm{D}=\mathrm{aL}^{\mathrm{n}},
$$

where $\mathrm{L}$ is the amplitude of the loudness stimulus (in millivolts), D the geometric mean duration said to match the loudness of that stimulus, and $a$ and $n$ parameters to be estimated, was fitted to the (untransformed) data of individual subjects and to the (transformed) pooled data, by a nonlinear least squares procedure.

Table 3 displays the results of this analysis. It can be seen that the fit of the power function to the data of individual subjects was similar in all cases to that to the pooled data. The pooled data are displayed in $\log -\log$ coordinates in Figure 1, in which the straight line represents the logarithmic form of the best fitting power function, $D=392 \mathrm{~L}^{.326}$. It can be seen in

Table 3

CMM Power Function Exponents

\begin{tabular}{clc}
\hline Subject & $\begin{array}{c}\text { Regression- } \\
\text { Estimated }\end{array}$ & $\begin{array}{c}\text { Range- } \\
\text { Estimated }\end{array}$ \\
\hline 1 & $.205 \pm .034^{*}$ & .226 \\
2 & $.492 \pm .059$ & .476 \\
3 & $.452 \pm .034$ & .460 \\
4 & $.379 \pm .023$ & .338 \\
5 & $.245 \pm .029$ & .235 \\
6 & $.237 \pm .019$ & .228 \\
7 & $.227 \pm .014$ & .255 \\
8 & $.338 \pm .027$ & .323 \\
9 & $.443 \pm .035$ & .397 \\
10 & $.260 \pm .021$ & .308 \\
Pooled & $.326 \pm .025$ & .325 \\
\hline
\end{tabular}

$* 1 S E$ 
Figure 1 that the psychophysical function displays a sinusoidal bowing. This is similar to that found for both category judgments and magnitude estimations of loudness (Ward, 1972, 1973).

The exponent of the best fitting power function is considerably (and statistically reliably, $\mathrm{t}=13.76$, $\mathrm{p}<.001)$ smaller than would be predicted from the aerage exponents of magnitude estimation experiments done on the two continua separately $\left(\mathrm{n}_{\boldsymbol{D}} \cong 1.0\right.$, $\mathrm{n}_{\mathrm{L}} \cong .67$, so $\mathrm{n}_{\mathrm{cmm}}=\mathrm{n}_{\mathrm{L}} / \mathrm{n}_{\mathrm{D}} \cong .67$ ). Cross (1973) has shown how sequential dependencies such as those reported above may bias such exponents. Cross (1973) represents magnitude estimates $\left(\mathrm{ME}_{\mathrm{ij}}\right)$ biased by sequential dependencies (first order only) as a power product of focal and preceding stimulus intensity $\left(S_{i}, S_{j}\right)$,

$$
M E_{i j}=a S_{i}^{m} S_{j}^{b},
$$

where a bias-free estimate of the correct exponent ( $n$ in Equation 2) is obtained from $n=m+b$. The exponent of the empirically best fitting power function of the form of Equation 2 is considered to be biased, and is represented by $m$ in Equation 3, not $n$ in Equation 2. Extending this analysis to cross-modality matches, in the present experiment, $m=.326$. Using multiple linear regression on the $S(N-K)$ by $S(N)$ matrix of log-geometric-mean responses, $b$ can be estimated, and for the present experiment $b=.016$. It is easily seen that $n=m+b=.342$ is still not near enough to $.67(\mathrm{t}=13.12, \mathrm{p}<.001)$, so the "regression effect" caused by sequential dependencies cannot explain the small exponent. In fact, if the analysis is extended to the stimuli further back in the sequence, bs of the order of .003 , but with negative sign, are discovered. Thus, in terms of their effect on the exponents, the cumulative contrast biases approximately cancel out the assimilative biases. It must be noted that both assimilative and contrast biases in the present experiment are very small in magnitude (although reliable statistically), and of the order of the smallest reported for magnitude estimation (Luce \& Green, 1974). Larger effects are expected in other types of experimental situations, where stimulus information is degraded.

A similar analysis was done on the $R(N-K)$ by $\mathrm{S}(\mathrm{N})$ matrix of log-geometric mean responses for the effects of the response "bin" on Trial N-1 only. For those data, $b_{r}$ (bias due to the response on Trial $N-1)$ was .024. This $b$ is somewhat larger than the first-order $\mathrm{b}$ for the stimulus analysis, indicating that the assimilation effect is probably associated with the previous response rather than the previous stimulus. This is contrary to the prediction of Luce and Green's (1974) model as it could be extended to cross-modality matches. However, by the same reasoning, the absence of a contrast effect in the

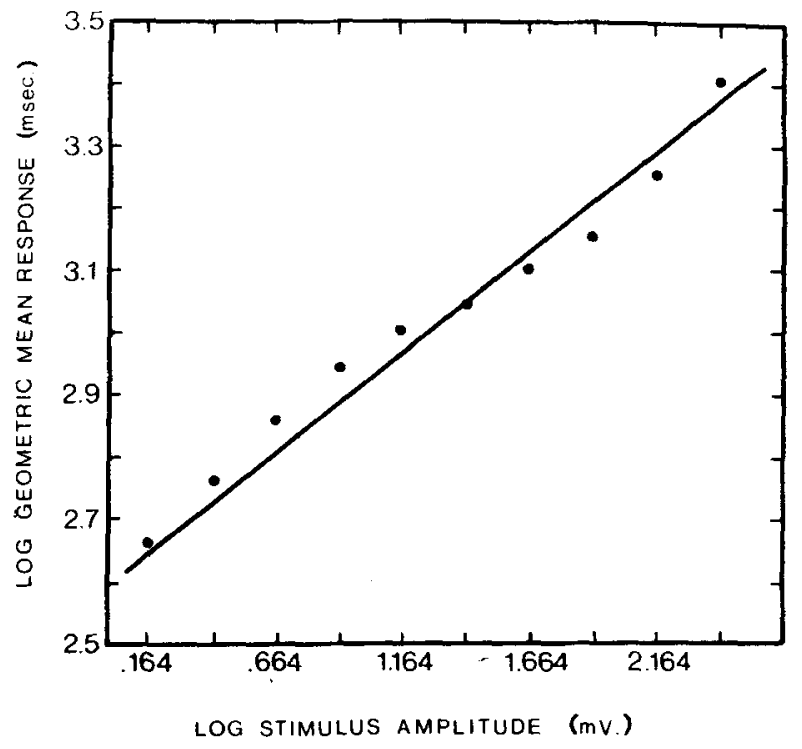

Figure 1. Log-log plot of the psychophysical function for the (transformed) pooled data. There are 500 observations per point (data of 10 subjects). The straight line is a graph of the best fitting power function, $D=392 \mathrm{~L} \cdot 326$, in $\log -\log$ coordinates.

response analysis and its presence in the stimulus analysis (coupled with the data of Ward and Lockhead, 1971) suggest that the contrast effect is more closely tied to previous stimuli, and may not reflect a tendency to use responses equally often.

Another way to view exponents of power functions has been suggested by Teghtsoonian (1971). He pointed out that the simple form of the power law (Equation 2 above) implies that

$$
\mathrm{n}=\log \mathrm{R}_{\mathrm{D}} / \log \mathrm{R}_{\mathrm{L}},
$$

where $R_{L}$ is the ratio of the largest to the smallest stimuli ( $\log R_{L}=2.24797$ in the present experiment), and $R_{\mathbf{D}}$ is the ratio of the corresponding average responses (geometric mean durations in the present experiment). Table 3 also shows the exponents of power functions calculated according to Equation 4 for the (untransformed) data of individual subjects and for the (transformed) pooled data. It can be seen that the agreement of the regression-estimated exponents and the range-estimated exponents is very good (the correlation between them is .965), indicating excellent power function fit. In fact, the pooled exponents are the same to two decimal places. Thus the exponents of the regression-fitted power functions were almost wholly determined by two factors: the range of stimuli presented (fixed by the experimenter and the same for every subject) and the range of responses chosen by subjects (different for every subject and free to vary within the constraints of the experimental situation). The average $\log R_{\mathbf{D}}$ chosen, .730, is in fact only about half as large as the 
average $\log \mathbf{R}_{\mathbf{D}}$ chosen in the magnitude estimation experiments which yielded an $n_{L}$ of .67 [estimated by Teghtsoonian (1971) to be 1.53]. This, of course, would explain, via Equation 4 and assuming $\log R_{L}$ to be relatively constant over experiments, why $n$ is only about half as large as would be predicted. Indeed, $\log R_{D}$ is similar in magnitude to log response ranges obtained_in category judgment of loudness experiments [Ward (1972) obtained an average $\log$ response range of .80 ]. If a category scale were being used in the present experiment, with labels equivalent to $1,2,3$, . . . etc., then a $\log R_{D}$ of .730 , which yields an $R_{D}$ of 5.4, represents about five to six categories. This corresponds to the average channel capacity for human subjects (Miller, 1956). In addition, it represents about the average number of different loudness stimuli subjects said they thought were presented.

\section{DISCUSSION}

Several formal theories of cross-modality matching have been proposed. Krantz (1972) discusses three of them, none of which can account for the sequential dependencies reported above and none of which make any predictions about the range of responses used. Luce and Green (1974) presented a detailed model of the magnitude estimation of loudness which does account for the first-order assimilation observed in those data in terms of a critical band of attention in their neural timing theory (cf. Luce \& Green, 1972). This model could be extended to the cross-modality matching situation, although complications would arise because of the nonnumeric response mode and the higher order contrast effects in the stimulus analysis. In addition, as mentioned earlier, if the assimilation effects are more strongly a function of previous responses than of previous stimuli, the Luce and Green model does not apply. For the present, then, it seems that the informal cognitive model proposed in the introduction provides at least as good an explanation of cross-modality matching data as do any of the other models, and in particular correctly predicted the existence of sequential dependencies in those data.

The cognitive model has a number of additional advantages when compared to the other models. First, it preserves the concept of matching continua, which binds together category judgment, magnitude estimation, and cross-modality matching, something few other models attempt. This matching process is conceived to be based on a single primitive operation, that of categorization, which can then be elaborated to conform to the rules of the experiment. This is quite similar to the cognitive theory of Baird (1970), with the exception that the categories of the present model are assumed to be at least ordinal, rather than nominal. They may, in fact, be interval scales, but that remains to be seen.

In addition, the matching process results in the equating of a response and stimulus continuum. If the matched continua are logarithmically transformed (as in the model of MacKay, 1963), then the matching process is described by Equation 4, which of course implies a power function relating the continua, with the exponent (n) a constant of proportionality determined by the relative $(\log )$ sizes of the two continua. This explains why power functions describe category judgments (Curtis, 1970; Marks, 1968; Ward, 1972) as well as magnitude estimations and cross-modality matches. And via Equation 4, specification of both response and stimulus ranges allows category judgments to be consistent with both magnitude estimations (Ward, 1973) and crossmodality matching.

A third problem avoided by the cognitive model is the transducer controversy. If power functions arise from equating dynamic ranges of stimulus continua to variously effected response ranges [as Teghtsoonian (1971) argues], then there need be no power function at the transducer. This would account for the successful scaling via magnitude estimation and cross-modality matching of various continua which have no single sensory transducer producing nerve impulses as a simple function of energy input (e.g., duration, length, distance, pain, electric shock, heinousness of crimes, etc.). All that is needed is a relatively smooth matching of one category scale to another. Especially appealing here is the interpretation of the exponent of a power function, not as an operating characteristic of a particular sensory transducer (although for some continua it may be a good approximation), but more generally in terms of the relative ranges of variously produced internal representations of aspects of the external world.

\section{REFERENCES}

BaIRD, J. C. A cognitive theory of psychophysics. I and II. Scandinavian Journal of Psychology, 1970, 11. 35-46 and 89-102.

Braida, L. D., \& Durlach, N. I. Intensity perception II. Resolution in one-interval patadigms. Joumal of the Acoustical Society of America, 1972. 51, 483-502.

Cross, D. V. Sequential dependencies and regression in psychophysical judgments. Perception \& Psychophysics, 1973, 14, 547-552.

CuRTIS, D. W. Magnitude estimations and category judgments of brightness and brightness intervals: A two-stage interpretation. Journal of Experimental Psychology. 1970, 83. 201-208.

KRANTZ, D. A theory of magnitude estimation and crossmodality matching. Journal of Mathematical Psychology, 1972, 9, 168-199. 
LIPETZ, L. E. The transter functions of sensory intensity in the nervous system. Vision Research. 1969, 9.1205-1234.

LUCE. R. D. \& GreEN. D. M. A neural timing theory for response times and the psychophysics of intensity. Psychological Review. 1972. 79. 14.57.

LcCE. R. D.. \& Green. D. M. The response ratio hypothesis for magnitude estimation. Journal of Mathematical Psychologs. 1974. 11. 1-14.

MACKAY. D. M. Psychophysics of perceived intensity: A theoretical basis for Fechner's and Stevens' laws. Science. 1963, 139. 1213-1216.

Marks. L. E. Stimulus range, number of categories. and form of the category scale. American Journal of Psychology, 1968, 81. $467.4 \% 9$.

Miller. G. A. The magical number seven plus or minus two: Some limits on our capacity for processing information. Psichological Review, 1956, 63, 81-97.

Simon, H. A. How big is a chunk? Science, 1974, 183, 482-488.

STEVENS. S. S. The psychophysics of sensory function. In W. Rosenblith (Ed.). Sensory communication. Cambridge: M.I.T. Press. 1961. Pp. 1-33.

Stevens. S. S. On the operation known as judgment. American Scientist. 1966, 54, 385.401.

Stevens. S. S. On predicting exponents for cross-modality matches. Perception \& Psychophysics. 1969, 6, 251-256.

Stevens. S. S. Neural events and the psychophysical law. Science, 1970. 170. 1043-1050.

Teghtsoonian. R. On the exponents in Stevens law and the constant in Ekman s law. Psychological Review, 1971. 78. 71-80.

WARD. L. M. Category judgments of loudnesses in the absence of an experimenter-induced identification function: Sequential effects and power function fit. Joumal of Experimental Psichologi. 1972. 94. 179-184.

WARD. L. M. Repeated magnitude estimations with a variable standard: Sequential effects and other properties. Perception \& Psichophysics. 1973. 14. 193-200.
W ARD. L. M.. \& LockheAD. G. R. Response system processes in absolute judgment. Perception \& Psychophysics. 1971, 9. 73-78. Woodrow. H. Time perception. In S. S. Stevens (Ed.). Handbook of experimental psychology. New York: Wiley, 1951. Pp. 1224-1236.

\section{NOTES}

1. Thanks are due to Wayne Kashinsky and Matthew Eisenberg who designed, built, and supplied the electronic timer.

2. Previous responses in Table 2 are actually "response blocks" which were obtained by "binning" the actual duration responses. Ten "bins" were created by inserting a bin boundary halfway between the (transformed) geometric mean responses of each subject to each adjacent pair of the 10 stimuli. Such "binning" is not totally satisfactory for the present purpose, but each response was used too infrequently to allow an analysis by actual responses. Other studies (e.g.. Braida \& Durlach. 1972) have found that creation of Thurstonian-type scales from magnitude estimations does not suffer from such binning. One other problem not totally alleviated by binning was an uneven distribution of entires in the $R(N-K)$ by $S(N)$ matrix of responses to $S(N)$. When there were no entries for a particular cell. the overall average response to that stimulus was inserted for purposes of the sequential dependencies analysis: this occurred for about $5-10$ of the 100 cells in each of the individual subject matrices. thus introducing a significant amount of error variance into those data. It was felt that the information gained from the analysis outweighed these flaws.

(Received for publication September 30. 1974: revision received May 27. 1975.) 